Understanding walking activity in multiple sclerosis: step count, walking intensity and uninterrupted walking activity duration related to degree of disability Peer-reviewed author version

NEVEN, An; Vanderstraeten, Annelien; JANSSENS, Davy; WETS, Geert \& FEYS, Peter (2016) Understanding walking activity in multiple sclerosis: step count, walking intensity and uninterrupted walking activity duration related to degree of disability. In: NEUROLOGICAL SCIENCES.

DOI: $10.1007 / \mathrm{s} 10072-016-2609-7$

Handle: http://hdl.handle.net/1942/21883 


\title{
STEP COUNT, WALKING INTENSITY AND UNINTERRUPTED WALKING ACTIVITY DURATION \\ RELATED TO DEGREE OF DISABILITY
}

\author{
An Neven, PhD ${ }^{1}$ \\ Annelien Vanderstraeten, MSc ${ }^{2}$ \\ Davy Janssens, $\mathrm{PhD}^{1}$ \\ Geert Wets, $\mathrm{PhD}^{1}$ \\ Peter Feys, $\mathrm{PhD}^{3}$
}

${ }^{1}$ IMOB Transportation Research Institute, Hasselt University, Diepenbeek, Belgium.

${ }^{2}$ Hasselt University, Diepenbeek, Belgium.

${ }^{3}$ REVAL Rehabilitation Research Institute, BIOMED Biomedical Research Institute, Faculty of Medicine and Life Sciences, Hasselt University, Diepenbeek, Belgium.

Corresponding author: An Neven, Transportation Research Institute (IMOB), Hasselt University, Wetenschapspark building 5 bus 6, 3590 Diepenbeek, Belgium.

Tel: +32(0)11269 130. Fax: +32(0)11269 199. Email: an.neven@uhasselt.be

\section{Concise title}

Walking activity in Multiple Sclerosis

\begin{abstract}
BACKGROUND: In Multiple Sclerosis (MS), physical activity (PA) is most commonly measured as number of steps, while also walking intensity and walking activity duration are keys for a healthy lifestyle.

OBJECTIVE: To investigate (1) the number of steps persons with MS (PwMS) take; (2) the number of steps they take at low and moderate intensity; and (3) their walking activity duration for 2, 3, 6, 10, 12 and 14 uninterrupted minutes; all related to the degree of disability.
\end{abstract}

METHODS: 64 PwMS participated, distinguished in a mild ( $\mathrm{n=31}$ ) and moderate MS subgroup ( $\mathrm{n}=34)$ based on their ambulatory dysfunction (Disease Steps). Standardized clinical tests were performed. Step data from the StepWatch Activity Monitor were collected for 7 consecutive days.

RESULTS: (1) Step count in PwMS was lower than PA recommendations, and is negatively influenced by a higher disability degree. (2) No walking was registered during $77 \%$ of the day. PwMS are making steps for $22 \%$ 
at low and only $1 \%$ at moderate intensity. (3) Both MS subgroups rarely walk for more than 6 uninterrupted minutes, especially not at moderate intensity.

CONCLUSIONS: PwMS need to be encouraged to make steps at moderate intensity, and to make steps for longer periods of time (minimal 10 uninterrupted minutes).

\section{Keywords}

Multiple Sclerosis, Walking, Physical Activity

\section{Acknowledgements}

The authors want to thank all PwMS for their voluntary participation.

\section{Declaration of Interest}

The authors report no conflicts of interest. The study was financially supported by a PhD fellowship from the Research council of Hasselt University (BOF-grant). 


\section{Introduction}

Persons with Multiple Sclerosis (PwMS), a progressive inflammatory and neurodegenerative disease of the central nervous system, are characterized by multiple neurological dysfunctions, amongst other motor impairments such as muscle weakness, hypertonia, ataxia as well as visual or cognitive dysfunction; all being considered as valuable bodily functions and impacting on gait [1-2]. Overall, one third of PwMS use an assistive mobility device [3]. Walking is a major problem in MS with $43 \%$ of the patients seeing this as a major problem that interferes in their life [4]. As a consequence, persons with physical limitations are showing decreased walking mobility and physical activity (PA) in a daily life community setting, although part of reduced activity is also related to disuse [5-6].

PA is defined as any bodily movement produced by skeletal muscles that requires energy expenditure. Physical inactivity, a failure to meet recommended levels of moderate-to-vigorous PA [7], has been identified as the fourth leading risk factor for global mortality in the general population. Also in PwMS, there is increased risk for secondary cardiovascular diseases and the metabolic syndrome [8]. Besides, it restricts participation in social life with an impact on travel behaviour, personal care, work and caring for family [3] . In general, regular PA at moderate intensity - such as walking, cycling or participating in sports - has significant benefits for health. Healthy adults should make 10.000 steps/day according to the public health recommendations [9], but PwMS have an average daily walking activity of 5.000-6.000 steps/day [10], with higher disability resulting in fewer steps [11]. Many PwMS seem to reduce their PA when diagnosed, despite the evidence that exercise is not leading to an increased relapse risk $[3,12]$ and potentially has a positive effect on fatigue, depression and quality of life (QoL) [13]. Activity monitoring devices such as pedometers are increasingly applied to measure PA in daily living [14], typically measuring the daily number of steps taken $[11,15]$.

However, not only the number of steps per day is important for a healthy lifestyle, but also the intensity and duration of walking activity needs to be high enough to reach health benefits from self-directed PA. Guidelines for PA in healthy subjects recommend 30 minutes of moderate intensity per day for 5 days/week, which may also be performed in periods of at least 10 minutes [9, 16-17]. Recent guidelines on PA in MS advocate that to achieve important fitness benefits, PwMS with mild to moderate disability need at least 30 minutes of moderate intensity aerobic activity 2 times/week and strength training exercises for major muscle groups 2 times/week [18]. The average step-rate cut-off for moderate intensity PA is generally agreed as 100 steps/minute [19-20], varying as a function of height, disease and disability [9]. Recently developed step-rate thresholds for PA intensity for ambulatory PwMS stated that for PwMS with minimal impairment and between a 
height of $158-190 \mathrm{~cm}$, the predicted step-rate at moderate-intensity threshold was between 83-104 steps/minute; and between 83-101 steps/minute for PwMS with mild-moderate impairment [21].

Despite the wealth of papers on step count in MS, there is little information whether the guidelines of moderate intensity are being reached in a community setting. It was shown previously that only a small proportion of PwMS (20\%) are achieving adequate amounts of daily moderate PA based on public health guidelines [22]. However, there was no information on the duration of uninterrupted walking activity at moderate intensity. Having knowledge about the duration when PwMS are making steps, is indispensable as PA guidelines recommend moderate intensity in periods of at least 10 uninterrupted minutes. Sedentary behaviour (i.e., any waking behaviour characterized by an energy expenditure of $\leq 1.5 \mathrm{MET}$ s while in a sitting of reclining posture [7]) is significant as well: when a person is sitting or lying, he/she is not spending time in higher intensity PA, contributing to a reduction in overall PA energy expenditure [23]). Therefore, it is important to have information about the duration when no walking is registered, which includes both sedentary behaviour, as well as periods of standing upright with no or very incidental movement.

The objectives of the present study are to investigate PA in subgroups of PwMS with different degree of disability by providing (1) the number of steps; (2) the number of steps at low and moderate intensity during the day; and (3) the walking activity duration for 2, 3, 6, 10, 12 and 14 uninterrupted minutes, in total as well as at low and moderate intensity .

\section{Methods}

\subsection{Participants}

The present study was part of a larger cross-sectional research project investigating changes in activity-related travel behaviour in PwMS. The study was approved by the ethical committees of Hasselt University, Rehabilitation and MS Center Overpelt, Antwerp University Hospital and National MS Centre Melsbroek. PwMS were recruited in the network of the REVAL Rehabilitation Research group, by neurologists of rehabilitation centers, and after information sessions in an MS-specialized fitness center and support groups of the MS Society Flanders. All PwMS gave written informed consent and participated voluntarily.

Participants were included when they made minimal one outdoor trip weekly without using a wheelchair (Disease Steps, DS < 6). PwMS were excluded if they were bedridden, or had a relapse or related corticosteroid treatment within one month before the study. PwMS were divided in two subgroups, according to their Disease Steps (DS) describing ambulatory dysfunction [24]. The DS is a clinical rating scale, based on a 
general physical examination by the researcher and the assistive devices needed to walk 25 feet. The DS is strongly correlated with the Expanded Disability Status Scale (EDSS) [25]. Persons in the 'mild' subgroup with DS $\leq 2$ experienced no to mild limitations or might have a visible abnormal gait, but did not require ambulation aids to walk 25 feet. Persons in the 'moderate' subgroup with DS 3-5 required intermittent or continuous unilateral or bilateral support to walk.

\subsection{Study design and outcome measures}

The study design was similar as in the larger research project and preceding pilot study [26]. At the first contact session, clinical measures were assessed and the StepWatch Activity Monitor (SAM) was thoroughly explained. During 7 consecutive days, walking activity was measured by wearing the SAM. The self-report indices and the SAM were returned in the second meeting.

\section{Descriptive clinical measures}

Clinical characteristics of PwMS by subgroup are shown in table 1. The Multiple Sclerosis Functional Composite (MSFC) measured the ambulation/leg function by the Timed 25-Foot Walk test (T25FW), the arm/hand function by the 9-Hole Peg test (9HPT) and cognition by the Paced Visual Serial Addition Test (PVSAT). The Multiple Sclerosis Walking Scale (MSWS-12) measured the impact of MS on walking ability, while the impact of fatigue on daily functioning was measured by the Modified Fatigue Impact Scale (MFIS) and health related QoL by the 36-item short-form health survey (SF-36).

Step count, walking intensity and walking activity duration

Walking activity was measured by the StepWatch Activity Monitor (SAM), a 2D accelerometer-based walking activity monitor, shown to be highly accurate in PwMS both at slow, comfortable and fastest speed [15]. The device is worn on the ankle, measures $75 \times 50 \times 20 \mathrm{~mm}$ and weighs 38 grams. The SAM continuously records the number of strides per time interval (steps taken by the leg monitored) over extended monitoring periods. Total recorded single-sided strides were doubled in this study to represent both left and right steps. The time interval of one minute is encouraged for standardization and also used in this study [27]. Main parameters of the SAM for the present study:

1. Step count: Number of steps per day. 
2. Walking intensity: Minutes and percentages of the day of no walking registered, low and moderate intensity. The cut-point for moderate intensity is based on the step-rate threshold for PwMS which varies between 83-104 steps/minute [21]. Therefore, we use cut-points 1-80 steps/minute for low intensity and $\geq 82$ steps/minute for moderate intensity. By 'no walking registered' we refer to both periods of sedentary behaviour and periods of standing upright with no or very incidental movement, that are not registered by the SAM.

3. Walking activity duration: Number of times PwMS are making steps for 2, 3, 6, 10, 12 and 14 uninterrupted minutes. Minutes are based on different walking tests in MS [28] and PA guidelines recommending 30 minutes of moderate intensity per day, which may be performed in bouts of at least 10 (uninterrupted) minutes [9, 16, 18].

\subsection{Data processing and statistical analysis}

Of the 108 PwMS of the larger research project, step data from the SAM were collected from 72 PwMS. Other participants were wheelchair bound $(n=13)$, did not receive a SAM due to operational reasons $(n=19)$, or were not willing to wear the SAM $(n=4)$. Step data of 5 complete days were used in this study (4 days for $n=4$ PwMS), hereby exceeding the threshold of 3 days needed to reach a reliability of 0.80 [29]. Eight participants were excluded because they did not fulfil this quality control, remaining 64 PwMS as participants. The dataset included both weekdays and weekend days for each participant.

Data were analysed using SPSS Statistics ( $\mathrm{p}$ 0.05). Descriptive analyses were used for the descriptive clinical measures. Regarding the SAM data, means were calculated per day. The Shapiro-Wilk test indicated non-normal distributions of most clinical measures and therefore, non-parametric Mann-Whitney and KruskalWallis tests for independent samples and Chi-Square tests were used to examine differences between subgroups.

\section{Results}

\subsection{Description of subgroups}

Descriptive clinical measures of PwMS by subgroup (mild $n=31$, moderate $n=33$ ) are shown in table 1 . The overall significant disparity of physical outcome measures among subgroups justified the selected cut-off score 2 of the DS. There were no significant differences for the cognitive outcome measures (PVSAT, cognitive component of MFIS, mental subscore of SF-36), suggesting that cognition is not differently affected. Age and disease characteristics were significant different between subgroups. 
INSERT TABLE 1

\subsection{Step count, walking intensity and walking activity duration}

\section{Step count}

The average total number of steps per day is presented in table 2 . The data show that in general, persons with mild MS make significant more steps each day than the moderate subgroup. The number of daily steps is significantly different between each DS separately (Kruskal-Wallis, $\mathrm{p}<0.01$ ), which is illustrated in figure 1.

\section{INSERT FIGURE 1}

\section{INSERT TABLE 2}

\section{Walking intensity}

The average number of steps at low and moderate intensity per day, as well as the average number of minutes (and percentage) at each intensity per day, is presented in table 2. PwMS in the mild subgroup are overall significant more active during the day regarding number of steps, and walk significant more minutes at both low and moderate intensity.

\section{Walking activity duration}

In table 2, the number of times that PwMS are making steps during an uninterrupted interval of $n$ (2-14) minutes per day is shown. There is a significant difference between both subgroups for the number of times they are making steps during 2 and 3 uninterrupted minutes, but not for the other longer time periods. The data show that both subgroups do not often walk during more than 6 uninterrupted minutes.

\subsection{Walking activity duration by intensity}

Table 3 presents the number of PwMS by subgroup that are making steps at different intensities during an uninterrupted interval of $\mathrm{n}(2-14)$ minutes. The results at low intensity for 2,3 and 6 minutes show that there is almost no difference between both subgroups. About $60-70 \%$ of PwMS are making steps at low intensity during 10 uninterrupted minutes at least once a day; but only about $30 \%$ are making steps during 14 uninterrupted 
minutes. There are only 6 PwMS in the mild subgroup (19\%) and 9 in the moderate subgroup (27\%) that are making steps 3 times per day for at least 10 uninterrupted minutes at low intensity (data not shown in the table).

Regarding the moderate intensity level, fewer PwMS of the moderate subgroup are making steps during 2-3 minutes at moderate intensity than the mild subgroup. Only $42 \%$ in the mild subgroup makes steps at moderate intensity for 6 uninterrupted minutes, decreasing to $24 \%$ in the moderate subgroup. The majority of PwMS in the moderate subgroup (64\%) never makes steps at moderate intensity, even not for short time periods. Only $13 \%$ and $3 \%$ of the mild and moderate subgroup walk $\geq 10$ minutes at moderate intensity.

\section{INSERT TABLE 3}

\section{Discussion}

The aim of this study was to investigate actual daily walking activity of PwMS, disentangled by number, intensity and duration of steps, under real-world conditions in relation to the disability level. It was not only found that the number of steps was reduced, proportional to the disability level, but also that a considerable amount of PwMS are not walking for an uninterrupted duration of 10 minutes, especially not at moderate intensity albeit capable. Results indicate a large non-compliance with PA recommendations advised for health promotion by both persons with mild and moderate MS.

The number of steps taken per day was previously shown to provide a reliable and valid outcome of free-living walking behavior in PwMS [30]. The number found in this study was comparable to previous studies[11, 31] while PwMS with mild ambulatory dysfunction made significant more steps than those with a higher disability degree $[11,32]$. The difference in daily step count can be partly explained by demographic factors as age [10], however, the impact of the latter considered to be small given only 8 years difference between subgroups.

To achieve important cardiovascular health benefits from PA, also the intensity and duration of walking needs to be high enough. The data demonstrate that PwMS of the mild subgroup clearly walk more minutes at moderate intensity than persons with moderate MS. Guidelines on PA advocate to have at least 30 minutes of moderate intensity activity, which may be performed in bouts of minimal 10 minutes. Therefore, the number of uninterrupted minutes that PwMS make steps at low and moderate intensities were analyzed as well. The results show that both MS subgroups, on average, do not often make steps during 10 uninterrupted minutes or more, and certainly not at moderate intensity levels. About 60-70\% of PwMS are making steps during 10 uninterrupted 
minutes at least once a day, but at low intensity; while only a quarter of PwMS made steps 3 times/day for 10 uninterrupted minutes at low intensity. Regarding the moderate intensity level, only $13 \%$ of PwMS in the mild subgroup made steps for an uninterrupted duration of 10 minutes at moderate intensity at least once a day, and only $3 \%$ of PwMS in the moderate subgroup. There was no single participant (of 64 PwMS) that made steps 3 times per day for 10 uninterrupted minutes at moderate intensity level.

The low frequency of PwMS walking for at least 10 minutes at moderate intensity, is not related to an incapacity to walk at moderate intensity in the mild subgroup, as $94 \%$ of PwMS in the mild subgroup were able to make steps at moderate intensity for 2 consecutive minutes. This implies that the majority of participants with mild ambulatory dysfunction of the present study are able to make steps for at moderate intensity, but simply don't do this for a longer period. It can be hypothesized that part of the patient sample may experience walkingrelated motor fatigue, leading to a slowing down of walking speed over time especially in the more disabled patient group. However, the latter cannot account for the large number of insufficiently active PwMS, and especially not in the mild subgroup where motor fatigue seems less prevalent. For future studies, one may include a long walking capacity test to be able to document the walking intensity capacity during prolonged uninterrupted walking.

Educational and motivational strategies are needed to encourage PwMS with mild ambulatory dysfunction to make steps at a higher intensity and during at least 10 uninterrupted minutes to improve their PA behaviour $[16,31]$. There should be a paradigm shift towards "PA for health", by which a more physically active lifestyle is promoted through behavioral and self-management interventions [33]. Pedometers and activity trackers can not only serve as an outcome measure for interventions but also as a direct information tool for patients, demonstrating the real type and intensity of daily PA performed by PwMS, including also outcomes of walking speed and duration besides the number of steps [34]. Besides, educational programs are needed to make patients 'exercise-ready' and enable the belief that an active healthy lifestyle can be achieved [35].

Overall, behavioral interventions can be effective for increasing and sustaining PA in PwMS [34, 36], and sometimes even leading to benefits in symptomatic outcomes like fatigue severity, depression and anxiety [37]. For other patients, tailored physical rehabilitation interventions may improve the walking activity in PwMS as well [38]. On a higher ICF level, improving the walking speed of PwMS appears to have important effects on the ability to participate in daily life, as it had a significant impact on health-related QoL and (assistance in) activities of daily living [39].

There are also limitations in the present study related to the measurement technology. The cut-point 
used for moderate intensity is based on the step-rate threshold for PwMS predicted by Agiovlasitis et al. [21], who conducted walking trials on a treadmill. As walking on a treadmill lead to a higher energy expenditure compared to overground walking [40], the real step-rate thresholds for classifying intensity during overground walking may actually be higher than the one used in our study. The approach on walking activity duration needs to be interpreted carefully as well. As the SAM uses time intervals of 1 minute for analysis in the StepWatch program, a minute is counted as active if a person makes minimal 1 (single-sided) step during this interval. Even if the person takes only a few steps in that minute, the SAM analysis counts this as walking for one minute at low intensity, hereby (incorrectly) assuming that the walking was continuous throughout that minute. Dall et al. [41] demonstrated that the step accumulation (i.e., the number of steps in a fixed period of time, like a 1 minute interval) and the cadence (i.e., the rate of stepping whilst walking, so only taking into account the amount of time spent stepping within that minute interval) are however not interchangeable outcome measures. It was shown that stepping in free-living is mostly composed of broken up 'household' type ambulation (e.g. movement about the home with a need to move around obstacles or a stop to perform tasks), rather than purposeful direct unhindered walking; as in their data set was found that only $12 \%$ of minutes with stepping was walked continuously during the whole minute [41]. Therefore, the activity designated as low intensity in our study may be overrated and a more correct designation of the parameter would therefore be 'step activity'. As well in the higher intensity levels, a proportion of the minutes may contain only a short duration of walking within that minute, making the actual cadence (intensity) higher than the average step accumulation that we used in the analysis, as our results don't fully adequately provide the time PwMS spend walking at a cadence above the threshold of 82 steps/min, However, our findings of walking/step activity duration at moderate intensity are considered as valid in the context of achieving health benefits from PA, as this implies that a person with MS is making steps (and performing PA) during several consecutive minutes without taking long rest breaks. Walking activity is an important part of PA, but there are also activities requiring a lot of body movements that are very intensive without foot displacement (e.g. gardening), and thus not measured by the SAM. There was no evidence (by making use of the SAM) to determine if the person was in a sitting or reclining posture, or had an energy expenditure of $\leq 1.5$ METs. It is possible that some minutes are classified as 'no walking registered', by which the person was standing upright, or possibly making weight shifts and taking small steps that were unregistered by the SAM. It was shown previously by Paul et al. [42] that these periods of incidental movement could account for up to four hours per day. Questionnaires such as the International PA questionnaires could be applied for this reason, however, have the drawback of potentially impaired recall to accurately quantify PA. 
The results of the present study show that most PwMS are not active enough to benefit from the positive health outcome effects of PA; both in terms of daily number of steps, intensity of walking and walking activity duration. Since the majority of PwMS with mild ambulatory dysfunction are able to make steps at moderate intensity for several consecutive minutes, these persons have to be encouraged to increase their uninterrupted walking activity in their community setting. 


\section{References}

1. Heesen C, Böhm J, Reich C, Kasper J, Goebel M, Gold SM. (2008). Patient perception of bodily functions in multiple sclerosis: gait and visual function are the most valuable. Mult Scler 7:988-991.

2. Balantrapu S, Sosnoff JJ, Pula JH, Sandroff BM, Motl RW. (2014). Leg spasticity and ambulation in multiple sclerosis. Mult Scler Int 2014:649390.

3. Larocca NG (2011). Impact of walking impairment in multiple sclerosis: perspectives of patients and care partners. Patient 3:189-201.

4. Pike J, Jones E, Rajagopalan K, Piercy J, Anderson P. (2012). Social and economic burden of walking and mobility problems in multiple sclerosis. BMC Neurology 12:94.

5. Motl RW, McAuley E, Snook EM (2005). Physical activity and multiple sclerosis: a meta-analysis. Mult Scler $11: 459-463$.

6. Pearson OR, Busse ME, van Deursen RW, Wiles CM. (2004). Quantification of walking mobility in neurological disorders.QJM 97:463-475.

7. Sedentary Behaviour Research Network. (2012). Letter to the editor: standardized use of the terms "sedentary" and "sedentary behaviours". Appl Physiol Nutr Metab 37:540-542.

8. Wens I, Dalgas U, Stenager E, Eijnde BO. (2013). Risk factors related to cardiovascular diseases and the metabolic syndrome in multiple sclerosis - a systematic review. Mult Scler 19:1556-1564.

9. Tudor-Locke C, et al. (2011). How many steps/day are enough? For older adults and special populations. Int J Behav Nutr Phy 8:80.

10. Dlugonski D, Pilutti A, Sandroff BM, Suh Y, Balantrapu S, Motl RW. (2013). Steps Per Day Among Persons With Multiple Sclerosis: Variation by Demographic, Clinical, and Device Characteristics. Arch Phys Med Rehab 94:1534-1539.

11. Gijbels D, Alders G, Van Hoof E, Charlier C, Roelants M, Broekmans T, Eijnde Bo, Feys P. (2010). Predicting habitual walking performance in multiple sclerosis: relevance of capacity and self-report measures. Mult Scler 16:618-626.

12. Tallner A, Waschbisch A, Wenny I, Schwab S, Hentschke C, Pfeifer K, Mäurer M. (2012). Multiple sclerosis relapses are not associated with exercise. Mult Scler 18:232-235.

13. Stroud NM, Minahan CL. (2009). The impact of regular physical activity on fatigue, depression and quality of life in persons with multiple sclerosis. Health Qual Life Out 7:86.

14. Weikert M, Motl RW, Suh Y, McAuley E, Wynn D. (2010). Accelerometry in persons with multiple sclerosis: Measurement of physical activity or walking mobility? J Neurol Sci 290:6-11.

15. Sandroff BM, Motl RW, Pilutti LA, Learmonth YC, Ensari I, Dlugonski D, Klaren RE, Balantrapu S, Riskin BJ. (2014). Accuracy of StepWatchTM and ActiGraph Accelerometers for Measuring Steps Taken among Persons with Multiple Sclerosis. PLoS One 9:e93511.

16. Tudor-Locke C. (2010). Steps to Better Cardiovascular Health - How Many Steps Does It Take to Achieve Good Health and How Confident Are We in This Number? Curr Cardiovasc Risk Rep 4:271-276.

17. Haskell WL, Lee IM, Pate RR, Powell KE, Blair SN, Franklin BA, Macera CA, Heath GW, Thompson PD, Bauman A. (2007). Physical Activity and Public Health. Updated Recommendation for Adults From the American College of Sports Medicine and the American Heart Association. Circulation 116:1081-1093. 
18. Latimer-Cheung AE, Martin Ginis KA, Hicks AL, Motl RW, Pilutti LA, Duggan M, Wheeler G, Persad R, Smith KM. (2013). Development of evidence-informed physical activity guidelines for adults with multiple sclerosis. Arch Phys Med Rehabil 94:1829-1836.

19. Marshall SJ, Levy SS, Tudor-Locke CE, Kolkhorst FW, Wooten KM, Ji M, Macera CA, Ainsworth BE. (2009). Translating physical activity recommendations into a pedometer-based step goal: 3000 steps in 30 minutes. Am J Prev Med 36:410-415.

20. Tudor-Locke C, Sisson SB, Collova T, Lee SM, Swan PD. (2005). Pedometer-determined step count guidelines for classifying walking intensity in a young ostensibly healthy population. Can J Appl Physiol 30:666-676.

21. Agiovlasitis S, Motl RW. (2014). Step-Rate Thresholds for Physical Activity Intensity in Persons With Multiple Sclerosis. Adapt Phys Act Q 31:4-18.

22. Klaren RE, Motl RW, Dlugonski D, Sandroff BM, Pilutti LA (2013). Objectively Quantified Physical Activity in Persons With Multiple Sclerosis. Arch Phys Med Rehabil 94:2342-2348.

23. Owen N, Healy GN, Matthews CE, Dunstan DW. (2010). Too Much Sitting: The Population-Health Science of Sedentary Behaviour. Exerc Sport Sci Rev 38:105-113.

24. Hohol MJ, Orav EJ, Weiner HL. (1995). Disease Steps in multiple sclerosis: A simple approach to evaluate disease progression. Neurology 45:251-255.

25. Hohol MJ, Orav EJ, Weiner HL. (1999). Disease steps in multiple sclerosis: a longitudinal study comparing disease steps and EDSS to evaluate disease progression. Mult Scler 5:349-354.

26. Neven A, Janssens D, Alders G, Wets G, Van Wijmeersch B, Feys P. (2013). Documenting outdoor activity and travel behaviour in persons with neurological conditions using travel diaries and GPS tracking technology: a pilot study in multiple sclerosis. Disabil Rehabil 35:1718-1725.

27. Mudge S, Stott NS. (2008). Test-retest reliability of the StepWatch Activity Monitor outputs in individuals with chronic stroke. Clin Rehabil 22:871-877.

28. Gijbels D, Eijnde BO, Feys P. (2011). Comparison of the 2- and 6-minute walk test in multiple sclerosis. Mult Scler 17:1269-1272.

29. Motl RW, Zhu W, Park Y, McAuley E, Scott JA, Snook EM. (2007). Reliability of scores from physical activity monitors in adults with multiple sclerosis. Adapt Phys Act Quart 24:245-253.

30. Motl RW, Pilutti LA, Learmonth YC, Goldman MD, Brown T. (2013). Clinical importance of steps taken per day among persons with multiple sclerosis. PLoS One 8:e73247.

31. Cavanaugh JT, Gappmaier VO, Dibble LE, Gappmaier E. (2011). Ambulatory Activity in Individuals with Multiple Sclerosis. J Neurol Phys Ther 35:26-33.

32. Filipovic GP, Grcic P, Matijaca M, Lusic I, Capkun V. (2011). Responsiveness of walking-based outcome measures after multiple sclerosis relapses following steroid pulses. Med Sci Monitor 17:CR704-10.

33. Motl RW. (2014). Lifestyle physical activity in persons with multiple sclerosis: the new kid on the MS block. Mult Scler 20:1025-1029.

34. Shammas L, Zentek T, von Haaren B, Schlesinger S, Hey S, Rashid A. (2014). Home-based system for physical activity monitoring in patients with multiple sclerosis (Pilot study). BioMed Eng OnLine 13:10. 
35. Feys P, Tytgat K, Gijbels D, De Groote L, Baert I, Van Asch P. (2013). Effects of an 1-day education program on physical functioning, activity and quality of life in community living persons with multiple sclerosis. NeuroRehabilitation 33:439-448.

36. Dlugonski D, Motl RW, Mohr DC, Sandroff BM. (2012). Internet-delivered behavioral intervention to increase physical activity in persons with multiple sclerosis: sustainability and secondary outcomes. Psychol Health Med 17:636-651.

37. Pilutti LA, Dlugonski D, Sandroff BM, Klaren R, Motl RW. (2014). Randomized controlled trial of a behavioral intervention targeting symptoms and physical activity in multiple sclerosis. Mult Scler 20:594-601.

38. Kalron A, et al. (2015). A personalized, intense physical rehabilitation program improves walking in people with multiple sclerosis presenting with different levels of disability: a retrospective cohort. BMC Neurology $15: 21$.

39. Goldman MD, Motl RW, Scagnelli J, Pula JH, Sosnoff JJ, Cadavid D. (2013). Clinically meaningful performance benchmarks in MS: timed 25-Foot Walk and the real world. Neurology 81:1856-1863.

40. Berryman N, Gayda M, Nigam A, Juneau M, Bherer L, Bosquet L. (2012). Comparison of the metabolic energy cost of overground and treadmill walking in older adults. Eur J Appl Physiol 112:1613-1620.

41. Dall PM, McCrorie PR, Granat MH, Stansfield BW. (2013). Step accumulation per minute epoch is not the same as cadence for free-living adults. Med Sci Sports Exerc 45:1995-2001.

42. Paul L, Rafferty D, Marshall-McKenna R, Gill JMR, McInnes I, Porter D, Woodburn J. (2014). Oxygen cost of walking, physical activity, and sedentary behaviours in rheumatoid arthritis. Scand J Rheumatol 43:28-34. 
Table 1: Clinical characteristics of PwMS by subgroup

\begin{tabular}{|c|c|c|c|c|}
\hline Outcome measure & $\begin{array}{c}\text { MS total }(n=64) \\
\text { DS 1-5 }\end{array}$ & $\begin{array}{l}\text { Mild MS }(n=31) \\
\text { DS 1-2 }\end{array}$ & $\begin{array}{c}\text { DS Moderate }(n=33) \\
\text { DS 3-5 }\end{array}$ & $\begin{array}{c}\text { p-value } \\
\text { comparison } \\
\text { between } \\
\text { subgroups }\end{array}$ \\
\hline Disease Steps (DS) & $2.8 \pm 1.5(1-5)$ & $1.5 \pm 0.5(1-2)$ & $4.1 \pm 0.8(3-5)$ & $<0.01$ \\
\hline $\begin{array}{l}\text { Type of MS } \\
\text { (RR/SP/PP/unknown) }\end{array}$ & $34 / 14 / 10 / 6$ & $26 / 0 / 3 / 2$ & $8 / 11 / 10 / 4$ & $<0.01$ \\
\hline Disease duration (years) & $13.6 \pm 8.8(2-38)$ & $11.5 \pm 8.4(2-30)$ & $15.6 \pm 8.8(2-38)$ & $<0.05$ \\
\hline Age (years) & $49.5 \pm 10.6(25-73)$ & $45.4 \pm 9.8(25-60)$ & $53.3 \pm 9.9(34-73)$ & $<0.01$ \\
\hline Gender (M/F) & $26 / 38$ & $9 / 22$ & $17 / 16$ & ns \\
\hline \multicolumn{5}{|l|}{ MS Functional Composite } \\
\hline Timed 25 Foot Walk (s) $\downarrow$ & $10.3 \pm 6.7(4.1-29.6)$ & $5.9 \pm 1.2(4.1-8.5)$ & $14.5 \pm 7.2(6.1-29.6)$ & $<0.01$ \\
\hline 9 Hole Peg Test R (s) $\downarrow$ & $25.7 \pm 7.4(15.2-49.9)$ & $22.9 \pm 4.9(15.2-38.1)$ & $28.4 \pm 8.5(16.2-49.9)$ & $<0.01$ \\
\hline 9 Hole Peg Test L (s) $\downarrow$ & $28.6 \pm 12.9(14.8-86.8)$ & $23.8 \pm 4.9(14.8-35.2)$ & $33.3 \pm 16.4(17.5-86.8)$ & $<0.01$ \\
\hline $\begin{array}{l}\text { Paced Visual Serial Addition } \\
\text { Test } \uparrow\end{array}$ & $48.8 \pm 12.2(0-60)$ & $51.2 \pm 9.6(26-60)$ & $46.6 \pm 13.9(0-60)$ & ns \\
\hline MS Walking Scale $12 \downarrow$ & $39.4 \pm 14.2(13-60)$ & $30.1 \pm 11.2(13-58)$ & $48.2 \pm 10.8(21-60)$ & $<0.01$ \\
\hline \multicolumn{5}{|l|}{ Modified Fatigue Impact Scale } \\
\hline Total MFIS $\downarrow$ & $39.0 \pm 15.8(3-74)$ & $34.1 \pm 17.0(3-74)$ & $43.7 \pm 13.7(13-65)$ & $<0.05$ \\
\hline Physical component $\downarrow$ & $19.7 \pm 7.6(1-32)$ & $16.6 \pm 8.0(1-32)$ & $22.6 \pm 5.9(5-32)$ & $<0.01$ \\
\hline Cognitive component $\downarrow$ & $15.5 \pm 8.4(2-34)$ & $14.3 \pm 8.0(2-34)$ & $16.5 \pm 8.8(2-31)$ & ns \\
\hline Psychosocial component $\downarrow$ & $4.1 \pm 3.6(0-28)$ & $3.2 \pm 2.1(0-8)$ & $4.8 \pm 4.50(0-28)$ & $<0.05$ \\
\hline \multicolumn{5}{|l|}{ Short Form (36) Health survey } \\
\hline SF36 physical subscore $\uparrow$ & $31.7 \pm 11.4(9.1-53.8)$ & $38.4 \pm 9.1(19.1-53.8)$ & $25.5 \pm 9.6(9.1-43.0)$ & $<0.01$ \\
\hline SF36 mental subscore $\uparrow$ & $49.6 \pm 11.8(20.2-66.4)$ & $50.5 \pm 10.0(27.7-66.1)$ & $48.8 \pm 13.3(20.2-66.4)$ & ns \\
\hline
\end{tabular}

Values are mean $\pm S D$ (range). Upward arrows indicate better performance with higher scores; downward arrows indicate worse performance with higher scores. Ns: not significant. P-value for Mann-Whitney \& Chi-Square comparison between MS subgroups. PwMS, Persons with Multiple Sclerosis; MS, Multiple Sclerosis; RR, Relapsing-Remitting; SP, Secondary Progressive; PP, Primary Progressive. 
Table 2: Step count, walking intensity and walking activity duration of PwMS by subgroup

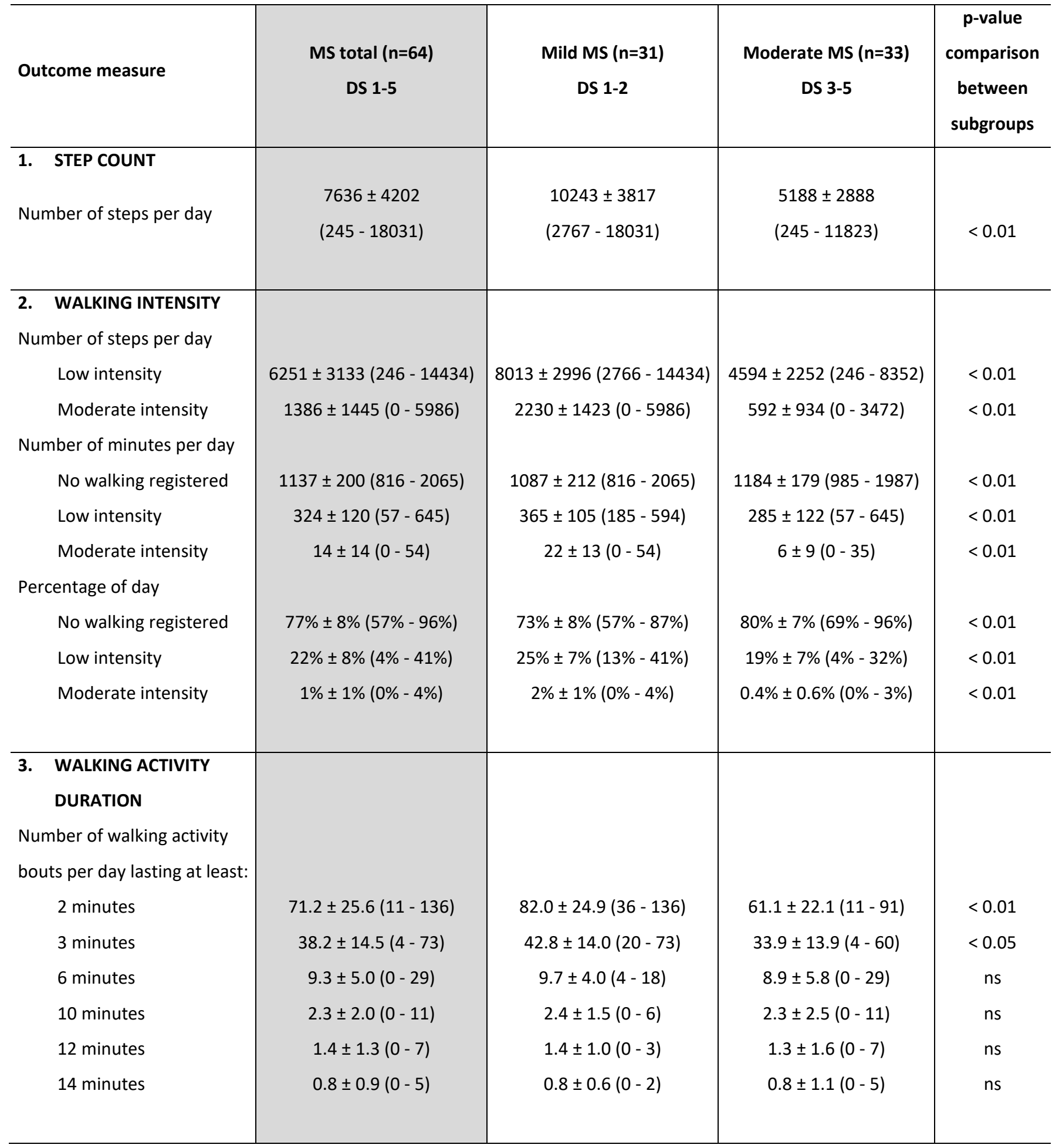

Values are mean \pm SD (range). Ns: not significant. P-value for Mann-Whitney comparison between MS subgroups. PwMS, Persons with Multiple Sclerosis; MS, Multiple Sclerosis.

Low intensity 1-80 steps/min; Moderate intensity $\geq 82$ steps/min. 
Table 3: Walking activity duration by intensity: number of PwMS by subgroup

\begin{tabular}{l|cc|cc}
\hline WALKING ACTIVITY & Low intensity & Moderate intensity \\
DURATION BY INTENSITY & $(1-80$ steps/min) & ( $\geq 82$ steps/min) \\
& & & & \\
\hline Mild MS subgroup ( $\mathrm{n}=31)$ & $\mathrm{n}$ & $\%$ & $\mathrm{n}$ & $\%$ \\
\hline 2 minutes & 31 & $100 \%$ & 29 & $94 \%$ \\
3 minutes & 31 & $100 \%$ & 26 & $84 \%$ \\
6 minutes & 31 & $100 \%$ & 13 & $42 \%$ \\
10 minutes & 23 & $74 \%$ & 4 & $13 \%$ \\
12 minutes & 16 & $52 \%$ & 2 & $6 \%$ \\
14 minutes & 10 & $32 \%$ & 1 & $3 \%$ \\
\hline Moderate MS subgroup $(\mathrm{n}=33)$ & $\mathrm{n}$ & $\%$ & $\mathrm{n}$ & $\%$ \\
\hline 2 minutes & 33 & $100 \%$ & 12 & $36 \%$ \\
3 minutes & 33 & $100 \%$ & 10 & $30 \%$ \\
6 minutes & 31 & $94 \%$ & 8 & $24 \%$ \\
10 minutes & 20 & $61 \%$ & 1 & $3 \%$ \\
12 minutes & 13 & $39 \%$ & 0 & $0 \%$ \\
14 minutes & 9 & $27 \%$ & 0 & $0 \%$ \\
\hline
\end{tabular}

